\title{
Returned Treatment Label Identifier Reference
}

National Cancer Institute

\section{Source}

National Cancer Institute. Returned Treatment Label Identifier Reference. NCI

Thesaurus. Code C87824.

A character or string used to name or characterize the reference identifier for a returned treatment label. 